\title{
DOPANT EFFECT ON PHENALENE BANDGAP CONTROL: A HÜCKEL MOLECULAR ORBITAL PERSPECTIVE
}

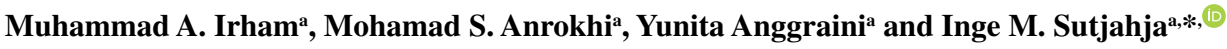 \\ aDepartment of Physics, Faculty of Mathematics and Natural Sciences, Institut Teknologi Bandung, 40132 Bandung, Indonesia
}

Recebido em 27/02/2021; aceito em 20/04/2021; publicado na web em 12/05/2021

\begin{abstract}
In this study, we investigate the effects of nitrogen and boron dopants on the properties of phenalene/phenalenyl systems based on the Hückel theory by using the Hueckel Molecular Orbital software. The dopants configurations are graphitic, pyridinic, and pyrrolic. The electronic configuration of bare phenalene confirms the delocalization of $\pi$ electrons and the radical properties of the molecule, which is in good agreement with the results of previous studies. Dopant types and positions strongly affect the number of $\pi$ electrons in the system, molecular orbital energy, total energy, average $\pi$-electron energy, and gap energy. The molecular energy level degeneracy strongly depends on the rotational symmetry of the system, in the order of graphitic, pyridinic, and pyrrolic. A preserved radical behavior and the number of $\pi$ electrons are found for the pyridinic dopant type, while closed electronic configuration is observed for graphitic and pyrrolic types. A lower gap energy is typically found for B-doped phenalene compared to that for N-doped phenalene; this opens the possibility for the enhancement of photoluminescence intensity. This study, although qualitative, confirms the effects of dopants on the chemical and physical properties of phenalene/phenalenyl systems.
\end{abstract}

Keywords: Hückel; phenalene; graphitic; pyridinic; pyrrolic.

\section{INTRODUCTION}

Graphene has gained considerable attention in recent years because of its unique properties. Graphene heteroatom doping has recently been extensively investigated to study the effect of doping on the structural, physicochemical, electrical, magnetic, and electrochemical properties of graphene. ${ }^{1-7}$ Precise and controlled n-type or p-type doping of graphene is a well-known strategy for engineering in the Fermi level to design new graphene-based materials with improved properties. Additionally, the electronic structure characteristics of these materials depend on not only the dopant concentration but also the doping symmetry pattern. Nitrogen and boron have gained interest as dopants because they are present in the same block as carbon in the periodic table, thus ensuring strong electron interaction with carbon atoms. In particular, the $2 p$ levels of boron and nitrogen are energetically close to those of the carbon atom, which facilitates the interaction within the $\pi$-electron system of the molecules and modification of their electronic properties. However, $\mathrm{B}$ and $\mathrm{N}$ have different electronegativity and number of valence electrons from $\mathrm{C}$; thus, they generally result in different effects. ${ }^{1}$ Each nitrogen and boron dopant can form three dopant configurations with graphene, namely, graphitic, pyridinic, and pyrrolic. ${ }^{2,3}$ In the development of photoluminescent materials, the proportion of pyridinic- $\mathrm{N}$ and pyrrolic- $\mathrm{N}$ to graphene quantum dots has a significant effect on photoluminescence intensity, ${ }^{2,3}$ because both configurations contribute differently to electron transition. Historically, molecules sharing honeycomb conformations of carbon atoms have been considered as graphene derivates. ${ }^{8}$

Phenalene $\left(\mathrm{C}_{13} \mathrm{H}_{10}\right)$ has gained considerable interest because of simple structure and low-cost fabrication as well as has derivatives with interesting properties and wide applications. ${ }^{4}$ Among the four isomers of phenalene, namely, $1 \mathrm{H}-, 2 \mathrm{H}-, 3 \mathrm{aH}-$, and $9 \mathrm{bH}$ - phenalenes, 1H-phenalene (shown in Figure 1(a)) is the only isomer synthesized to date and is considered to be the most stable. ${ }^{5}$ However, in all these isomers, the loss of the hydrogen moiety results in the formation of a

*e-mail: inge@fi.itb.ac.id phenalenyl system, $\mathrm{C}_{13} \mathrm{H}_{9}$. The phenalenyl radical is an odd alternant hydrocarbon radical with high stability and amphoteric redox nature. This molecule has long been studied as a model to understand the fundamental aspects such as chemical reactivity, stability, and physical properties of polycyclic aromatic hydrocarbon radicals to enhance the functionalities of the hydrocarbon system. ${ }^{6,7}$ The recent experimental study results show that a phenalenyl radical $/ 1 \mathrm{H}$ phenalene system can be an effective catalyst for the formation of interstellar $\mathrm{H}_{2} .{ }^{9}$ A $13-\pi$ phenalenyl neutral radical or ionic species, namely, a $12-\pi$ electron phenalenyl cation and a $14-\pi$ electron anion, is an aromatic system because they sustain well-defined diatropic perimeter ring currents. ${ }^{10}$ The molecule is commonly depicted by the delocalized $12 \pi$-electron perimeter surrounding the central carbon atom because of the strong resonance stabilization of the phenalenyl radical, as shown in Figure 1(b). The previously mentioned molecules have been explored for the development of new conjugated electronic systems with intriguing electronical and magnetic properties. ${ }^{11,12}$ The replacement of the central carbon atom of the phenalenyl motif with a $\mathrm{B}\left(\mathrm{C}_{12} \mathrm{BH}_{9}\right)$ or $\mathrm{N}$ atom $\left(\mathrm{C}_{12} \mathrm{NH}_{9}\right)$ is predicted to sustain strong paratropic perimeter ring currents, which indicates antiaromaticity- $\mathrm{a}$ magnetic criterion. It was noted that diatropic and paratropic ring currents represent anticlockwise and clockwise circulations, respectively, in current-density maps under a perpendicular external magnetic field of $\pi$-electron systems. ${ }^{13}$<smiles>CC1C=Cc2cccc3cccc1c23</smiles>

(a)<smiles>C1=CC=C2C=CC=C3C=CC=C(C=1)C23</smiles>

(b)
Figure 1. (a) Phenalene structure $\left(C_{13} H_{10}\right)$ and (b) depiction of the phenalenyl system ${ }^{5}$

Recently, Salinas ${ }^{8}$ reported that the selective N (electronaccepting) and $\mathrm{B}$ (electron-donating) substitution of the central carbon 
in phenalenyl and its derivatives modifies the number of $\pi$-electrons of the molecule, altering the frontier orbital and changing the electronic and magnetic properties. Moreover, because the $2 p_{z}$ orbital of boron is higher (lower for nitrogen) in energy that of carbon, a certain frontier $\pi$ molecular orbital of the central carbon atom (that belongs to the $A_{2}{ }^{\prime \prime}$ irreducible representation) is destabilized (stabilized for nitrogen), and its shifting might eventually result in small highest occupied molecular orbital (HOMO)-lowest unoccupied molecular orbital (LUMO) energy gaps. In the case of photoluminescence, a narrower bandgap due to the modification of the $\pi$-type orbital could increase excitation probabilities, thus increasing photoluminescence intensity. This study focused on simple boron and nitrogen dopants for phenalene/phenalenyl with varying dopant position, to probe the possible effects related to the change in the molecular energy level and gap energy.

Currently, there are many computational approaches to study the molecular energy of graphene-based molecules. However, approaches based on advanced calculations might be relatively hard to perform, and the obtained information might be too difficult for undergraduate students to understand. The Hückel molecular orbital (HMO) theory is a well-known approach for $\pi$ molecular orbital calculations and clearly and simply elucidates the mechanisms and structures of $\pi$-electron systems. Moreover, the calculation of the $\pi$ molecular orbital facilitates the prediction of the optical gap of a molecule on the basis of the experimentally measured fundamental gap. ${ }^{14,15}$ Herein, we present the $\pi$ molecular orbital calculation of phenalene and its doped molecule by using the HMO theory via the HMO software, to predict the $\pi$ electron system behavior and the optical gap.

\section{METHODS}

The HMO theory, introduced by Hückel in $1931,{ }^{16-19}$ is an old calculation method that provides groundbreaking foundation in understanding the molecular orbital of a system (See Ref..$^{20}$ for a complete review). The calculation offered by the Hückel method is very simple and feasible for students with basic chemistry and quantum physics knowledge. Although the assumption of the theory is not very precise, its implementation succeeded in predicting many phenomena in various fields and is being continuously developed by either relating to the Hückel theory itself or to other theories and methods. ${ }^{21}$

The following equation depicts the Hückel theory based on the Schrödinger equation for a $\pi$ electron system:

$$
\hat{H} \Psi=E \Psi
$$

Within the linear combination of an atomic orbital LCAO model, ${ }^{20}$ the $\pi$ molecular orbital, $\Psi$, can be written as a linear combination of atomic orbital $\phi$,

$$
\Psi_{n}=\Sigma_{i} c_{n, i} \phi=c_{n, 1} \phi_{1}+c_{n, 2} \phi_{2}+c_{n, 3} \phi_{3}+\ldots+c_{n, 13} \phi_{13}
$$

where $c_{n, i}$ is the coefficient of linear combination, and index $i(i=1$, $2, \ldots$.$) is applicable for all carbon atom constituting the molecule.$ Hence, the Hamiltonian matrix element according to the Hückel theory is

$$
H_{i j}=\phi_{i}|\hat{H}| \phi_{j}=\left\{\begin{array}{l}
\alpha, \quad i=j \\
\beta, i-j= \pm 1
\end{array}\right.
$$

where $\alpha$ and $\beta$ are the Coulomb integral and resonance integral of the $\pi$ electron system, respectively. For a carbon atom, $\alpha\left(=\alpha_{C}\right)$ is the core energy of an electron localized to the $2 p$ atomic orbital, and
$\beta\left(=\beta_{\mathrm{CC}}\right)$ is the energy associated with the interaction of two carbon $2 p$ orbitals overlapping in a $\pi$ (parallel) fashion at the $\mathrm{C}-\mathrm{C}$ bond. The overlap integral within the Hückel theory is defined as

$$
S_{i j}=\left\langle\phi_{i} \mid \phi_{j}\right\rangle=\delta_{i j}
$$

Further, the $\pi$-bond formation energy of a system can be calculated by comparing the total energy of the $\pi$ electron system in the molecule and the isolated system as follows:

$$
E_{\pi-\text { bond (formation) }}=E_{\text {total }, \pi}-\mathrm{E}_{\text {isolated }}
$$

Finally, the gap energy was calculated as follows:

$$
E_{\text {gap }}=E_{\text {LUMO }}-E_{\text {Номо }}
$$

The bond order between different atoms $i$ and $j$ can be calculated as

$$
\rho_{i j}=n_{e} \sum_{i, j=1} c_{i} c_{j}
$$

where $c_{i}$ is the coefficient of the atomic orbital linear combination, and $n_{e}$ is the occupation number of $\pi$ electrons in a certain orbital molecule.

The HMO software ${ }^{22}$ is a free, interactive, and simple software that performs Hückel-theory-based calculations on the basis of the molecular structure of the input system. Figure 2 shows the HMO software interface. The program automatically constructs the secular determinant from the molecular structure of the input system and computes eigenvectors and eigenvalues by the Jacobi diagonalization method. This program can accommodate various heteroatoms in the calculation, and the output of the program can be displayed in a graphic or tabular form.

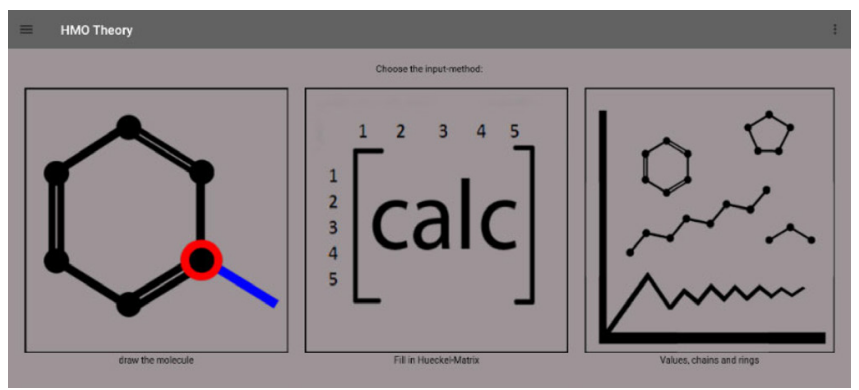

Figure 2. HMO software interface ${ }^{22}$

In the results of this software, carbon atom bonding with hydrogen is considered to be implicit, while bonding between boron and/or nitrogen and hydrogen is not shown.

The values of $\alpha$ and $\beta$ in equation (3) depend on the atom type and coordination number. These are related to the different core energies of heteroatom $X$ and the change in the effective electronegativity of the remaining nonbonded $p$ orbitals at the center.

$$
\begin{gathered}
\alpha_{X}=\alpha+h_{X}|\beta| \\
\beta_{X Y}=k_{X Y}|\beta|
\end{gathered}
$$

Table 1 lists the common values of $h_{X}$ and $k_{X Y}$ for $\mathrm{C}, \mathrm{N}$, and B atoms. ${ }^{23}$ The opposite values of $h_{X}$ for $\mathrm{N}$ and $\mathrm{B}$ arise because of the respective higher and lower electronegativities compared to that of $\mathrm{C}$.

Notably $h_{X}$ and $k_{X Y}$ for $\mathrm{N}$ and B input in the HMO software can be set to specific values or varied based on experimental UV spectral data. $^{22}$ 
Table 1. Values of $h_{X}$ and $k_{X Y}$ for C, N, and B through Hückel parametrization ${ }^{23}$

\begin{tabular}{ccc}
\hline Element & $h_{X}$ & $k_{X Y}$ \\
\hline $\mathrm{C}$ & $h_{C}=0.00$ & $k_{C C}=-1.00$ \\
$\mathrm{~N} 2 *$ & $h_{N}=-0.51$ & $k_{N C}=-1.02$ \\
$\mathrm{~N} 3 * *$ & $h_{N}=-1.37$ & $k_{N C}=-0.89$ \\
$\mathrm{~B}$ & $h_{B}=0.45$ & $k_{B C}=-0.73$ \\
\hline
\end{tabular}

* Dicoordinated. ** Tricoordinated, planar geometry.

\section{RESULTS AND DISCUSSION}

\section{Basic structure and energy level of phenalene}

The phenalene structure and each carbon atom is numbered for the HMO software input, as shown in Figure 3. Through Hückel parametrization, its determinant Hamiltonian matrix is evaluated, as summarized in Table 2, in which $x$ denotes $(\alpha-\varepsilon) / \beta$, where $\varepsilon$ is the molecular orbital eigen value (Figure 4).

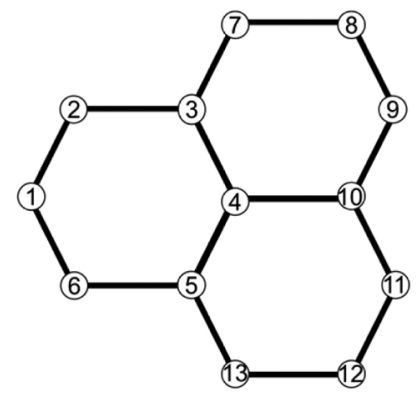

Figure 3. Structure of phenalene with carbon atom numbering

The orbital energy of phenalene is shown in Figure 4, along with the molecular orbital, while the molecular orbital coefficient is shown in Table 3. The obtained energy levels well-match those reported in a previous study ${ }^{7}$ for phenalenyl. The small difference in the energy magnitude might be due to the different number of hydrogen atoms, which is not sensitive in HMO software. Figure 4 shows that the lowest energy is non-degenerate, while the next energy levels are two-fold degenerate and three-fold degenerate. The electrons fully occupy these energy levels with opposite spins to fulfill the Pauli restriction. The $\mathrm{HOMO}$ with single electron occupation is undegenerate and is also called the singly occupied molecular orbital (SOMO). The occurrence of the SOMO confirms the radical character of phenalene, which is consistent with the results of previous studies. ${ }^{6,7}$ The total energy of the phenalene system is $E_{\pi}=13 \alpha+17.826 \beta$, which corresponds to one $\pi$ electron, $1.371 \beta$. The obtained bare phenalene gap energy is $1.000 \beta$. From the molecular orbital perspective, the lowest energy related to the maximum overlap of an atomic orbital. The number of nodal lines increases with increasing energy.

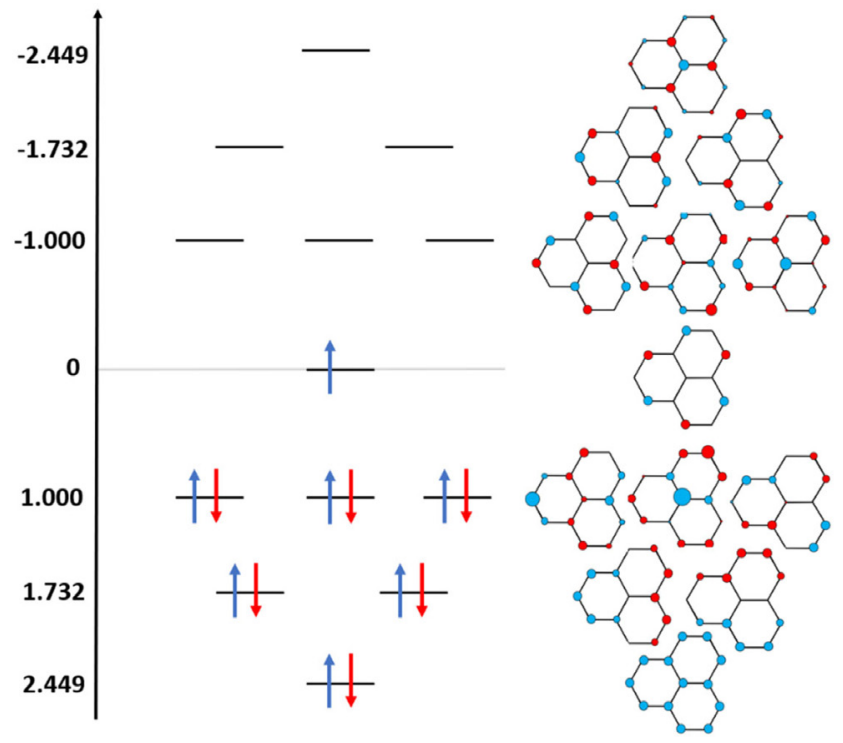

Figure 4. Calculated energy level of phenalene and its molecular orbital. Left: arrows with different colors represent the spin-up and spin-down electrons. Right: blue and red filled circles represent the positive and negative signs for the linear combination coefficient in the molecular orbital, respectively (Table 3)

The phenalene bond order calculation results is shown in Figure 5. From this figure, one can see that a high-symmetry pattern is observed, and the bond order at the far edge from the center of phenalene is higher than at the center. These results agree with the delocalized structure of $12 \pi$-electrons surrounding a central carbon atom (Figure 1(b)).

\section{Dopant effect on the energy level of phenalene}

Nitrogen and boron dopant sites on phenalene consist of graphitic,

Table 2. Determinants of phenalene Hamiltonian matrix through Hückel parametrization

\begin{tabular}{cccccccccccccccc}
\hline & $1 \mathrm{C}$ & $2 \mathrm{C}$ & $3 \mathrm{C}$ & $4 \mathrm{C}$ & $5 \mathrm{C}$ & $6 \mathrm{C}$ & $7 \mathrm{C}$ & $8 \mathrm{C}$ & $9 \mathrm{C}$ & $10 \mathrm{C}$ & $11 \mathrm{C}$ & $12 \mathrm{C}$ & $13 \mathrm{C}$ \\
\hline $1 \mathrm{C}$ & $-\mathrm{x}$ & 1.0 & 0.0 & 0.0 & 0.0 & 1.0 & 0.0 & 0.0 & 0.0 & 0.0 & 0.0 & 0.0 & 0.0 \\
$2 \mathrm{C}$ & 1.0 & $-\mathrm{x}$ & 1.0 & 0.0 & 0.0 & 0.0 & 0.0 & 0.0 & 0.0 & 0.0 & 0.0 & 0.0 & 0.0 \\
$3 \mathrm{C}$ & 0.0 & 1.0 & $-\mathrm{x}$ & 1.0 & 0.0 & 0.0 & 1.0 & 0.0 & 0.0 & 0.0 & 0.0 & 0.0 & 0.0 \\
$4 \mathrm{C}$ & 0.0 & 0.0 & 1.0 & $-\mathrm{x}$ & 1.0 & 0.0 & 0.0 & 0.0 & 0.0 & 1.0 & 0.0 & 0.0 & 0.0 & 0.0 & 0 \\
$5 \mathrm{C}$ & 0.0 & 0.0 & 0.0 & 1.0 & $-\mathrm{x}$ & 1.0 & 0.0 & 0.0 & 0.0 & 0.0 & 0.0 & 0.0 & 1.0 \\
$6 \mathrm{C}$ & 1.0 & 0.0 & 0.0 & 0.0 & 1.0 & $-\mathrm{x}$ & 0.0 & 0.0 & 0.0 & 0.0 & 0.0 & 0.0 & 0.0 \\
$7 \mathrm{C}$ & 0.0 & 0.0 & 1.0 & 0.0 & 0.0 & 0.0 & $-\mathrm{x}$ & 1.0 & 0.0 & 0.0 & 0.0 & 0.0 & 0.0 \\
$8 \mathrm{C}$ & 0.0 & 0.0 & 0.0 & 0.0 & 0.0 & 0.0 & 1.0 & $-\mathrm{x}$ & 1.0 & 0.0 & 0.0 & 0.0 & 0.0 \\
$9 \mathrm{C}$ & 0.0 & 0.0 & 0.0 & 0.0 & 0.0 & 0.0 & 0.0 & 1.0 & $-\mathrm{x}$ & 1.0 & 0.0 & 0.0 & 0.0 \\
$10 \mathrm{C}$ & 0.0 & 0.0 & 0.0 & 1.0 & 0.0 & 0.0 & 0.0 & 0.0 & 1.0 & $-\mathrm{x}$ & 1.0 & 0.0 & 0.0 \\
$11 \mathrm{C}$ & 0.0 & 0.0 & 0.0 & 0.0 & 0.0 & 0.0 & 0.0 & 0.0 & 0.0 & 1.0 & $-\mathrm{x}$ & 1.0 & 0.0 \\
$12 \mathrm{C}$ & 0.0 & 0.0 & 0.0 & 0.0 & 0.0 & 0.0 & 0.0 & 0.0 & 0.0 & 0.0 & 1.0 & $-\mathrm{x}$ & 1.0 \\
$13 \mathrm{C}$ & 0.0 & 0.0 & 0.0 & 0.0 & 1.0 & 0.0 & 0.0 & 0.0 & 0.0 & 0.0 & 0.0 & 1.0 & $-\mathrm{x}$ \\
\hline
\end{tabular}


Table 3. Hückel coefficient, $c_{i}$, values for molecular orbital, $\Psi$, determination

\begin{tabular}{|c|c|c|c|c|c|c|c|c|c|c|c|c|c|}
\hline & $\Psi_{1}$ & $\Psi_{2}$ & $\Psi_{3}$ & $\Psi_{4}$ & $\Psi_{5}$ & $\Psi_{6}$ & $\Psi_{7}$ & $\Psi_{8}$ & $\Psi_{9}$ & $\Psi_{10}$ & $\Psi_{11}$ & $\Psi_{12}$ & $\Psi_{13}$ \\
\hline$c_{1}$ & 0.183 & 0.408 & 0.003 & -0.480 & -0.249 & 0.086 & 0.0 & -0.312 & 0.142 & 0.427 & 0.408 & -0.020 & -0.183 \\
\hline$c_{2}$ & 0.224 & 0.355 & -0.201 & 0.202 & -0.076 & 0.391 & -0.408 & 0.365 & 0.208 & -0.154 & -0.363 & -0.186 & 0.224 \\
\hline$c_{3}$ & 0.365 & 0.207 & -0.352 & -0.278 & 0.172 & 0.305 & 0.0 & -0.053 & -0.350 & -0.273 & 0.222 & 0.343 & -0.365 \\
\hline$c_{4}$ & 0.447 & 0.0 & 0.0 & -0.129 & 0.525 & -0.086 & 0.0 & 0.026 & -0.132 & 0.531 & 0.0 & 0.0 & 0.447 \\
\hline$c_{5}$ & 0.365 & 0.201 & 0.355 & -0.202 & 0.076 & -0.391 & 0.0 & 0.365 & 0.208 & -0.154 & 0.186 & -0.363 & -0.365 \\
\hline$c_{6}$ & 0.224 & 0.352 & 0.207 & 0.278 & -0.172 & -0.305 & 0.408 & -0.053 & -0.350 & -0.273 & -0.343 & 0.222 & 0.224 \\
\hline$c_{7}$ & 0.224 & 0.003 & -0.408 & -0.351 & -0.277 & 0.0 & 0.408 & -0.338 & 0.274 & -0.104 & -0.020 & -0.408 & 0.224 \\
\hline$c_{8}$ & 0.183 & -0.201 & -0.355 & -0.074 & -0.449 & -0.305 & 0.0 & 0.390 & 0.076 & 0.377 & -0.186 & 0.363 & -0.183 \\
\hline$c_{9}$ & 0.224 & -0.352 & -0.207 & 0.278 & -0.172 & -0.305 & -0.408 & -0.053 & -0.350 & -0.273 & 0.343 & -0.222 & 0.224 \\
\hline$c_{10}$ & 0.365 & -0.408 & -0.003 & 0.351 & 0.277 & 0.0 & 0.0 & -0.338 & 0.274 & -0.104 & -0.408 & 0.02 & -0.365 \\
\hline$c_{11}$ & 0.224 & -0.355 & 0.201 & 0.202 & -0.076 & 0.391 & 0.408 & 0.365 & 0.208 & -0.154 & 0.363 & 0.186 & 0.224 \\
\hline$c_{12}$ & 0.183 & -0.207 & 0.352 & -0.149 & -0.353 & 0.391 & 0.0 & -0.027 & -0.482 & 0.258 & -0.222 & -0.343 & -0.183 \\
\hline$c_{13}$ & 0.224 & -0.003 & 0.408 & -0.351 & -0.277 & 0.0 & -0.408 & -0.338 & 0.274 & -0.104 & 0.020 & 0.408 & 0.224 \\
\hline$\sum c_{i}^{2}$ & 1.0 & 1.0 & 1.0 & 1.0 & 1.0 & 1.0 & 1.0 & 1.0 & 1.0 & 1.0 & 1.0 & 1.0 & 1.0 \\
\hline
\end{tabular}

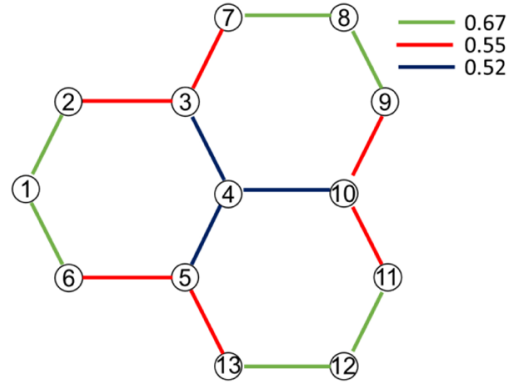

Figure 5. Bond order of phenalene; different colors refer to different bond order values

pyridinic, and pyrrolic types, as shown in Figure 6. The coordination number is three for the graphitic type of the dopant, while two for the pyridinic and pyrrolic type of dopants.

Figure 7 shows the energy level of the different sites of nitrogendoped phenalene and boron-doped phenalene, along with the electron configuration on each orbital molecule and orbital molecule for the SOMO level. Notably, different values of $\alpha$ and $\beta$ induce a small change in the energy values. It can be observed that changing dopants generally cause a change in the number of $\pi$ electrons and the energy level diagram

$\mathrm{N}$-pyridinic, B-pyridinic, N-pyrrolic, and B-pyrrolic phenalene dopant types donate $1 \pi$-electron to the phenalene molecule; the $\mathrm{N}$-graphitic type donates $2 \pi$-electrons; and no $\pi$-electron is donated by the B-graphitic type to phenalene. Among the three types of dopant position, the graphitic type of dopant resulted in the maximum energy level degeneracy, followed by the pyridinic type, and no degeneracy for the pyrrolic-type dopant. In relation to the symmetry rotation of the system, the graphitic-type dopant is highly symmetric. In contrast, the pyrrolic-type dopant changes the honeycomb pattern of the system; thus, its symmetric rotation is broken and no degeneracy of the energy level is observed. Moreover, the three degeneracies near the SUMO level decreased into two as the number of symmetry rotations decreased. ${ }^{24}$ From the stability perspective, the pyridinic-type dopant, either for nitrogen or boron, still confers the radical character to the electron, making the doped molecule reactive, similar to the case of bare phenalene. Additionally, the existence of an unpaired electron could enhance the magnetic properties of the system.

The number of $\pi$ electrons, total energy, average $\pi$-electron energy, and gap energy for bare phenalene and its doped molecules are summarized in Table 4 . For the $\mathrm{N}$ dopant, different $\alpha$ and $\beta$ values reveal different energy values, which is not the case for the B dopant.

Table 4 shows that the total energy of the molecule sensitivity depends on the $\alpha$ and $\beta$ values. However, in terms of the $\beta$ parameter, the average energy of $\pi$ electrons is higher for $\mathrm{N}$-doped than that for B-doped phenalene. Moreover, the energy gap firmly depends on the type and dopant position. For both $\mathrm{N}$-doped and B-doped phenalene, the gap energy decreases in magnitude for pyridinic, pyrrolic, and graphitic types. In general, boron doping results in lower gap energy than nitrogen doping, in agreement with a recent study by Cyrański. ${ }^{13}$ A lower energy gap facilitates a higher photoluminescence intensity as the electron gets easier to excite to the LUMO. This study suggests that controlling the dopant type and position for heteroatom-doped phenalene systems facilitates tailoring their structure-property

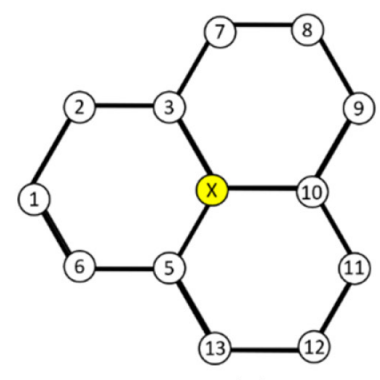

(a)

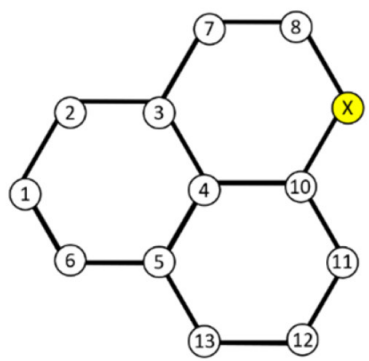

(b)

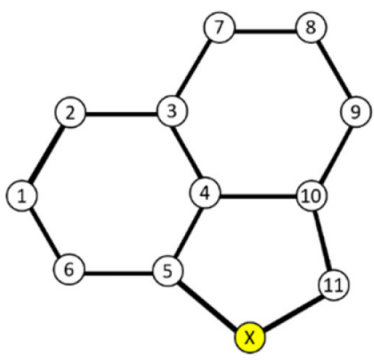

(c)

Figure 6. (a) Positions of graphitic, (b) pyridinic, and (c) pyrrolic types of the dopant on phenalene. The yellow circle represents the heteroatom (where $X=$ $N$ or $B$ atom) 

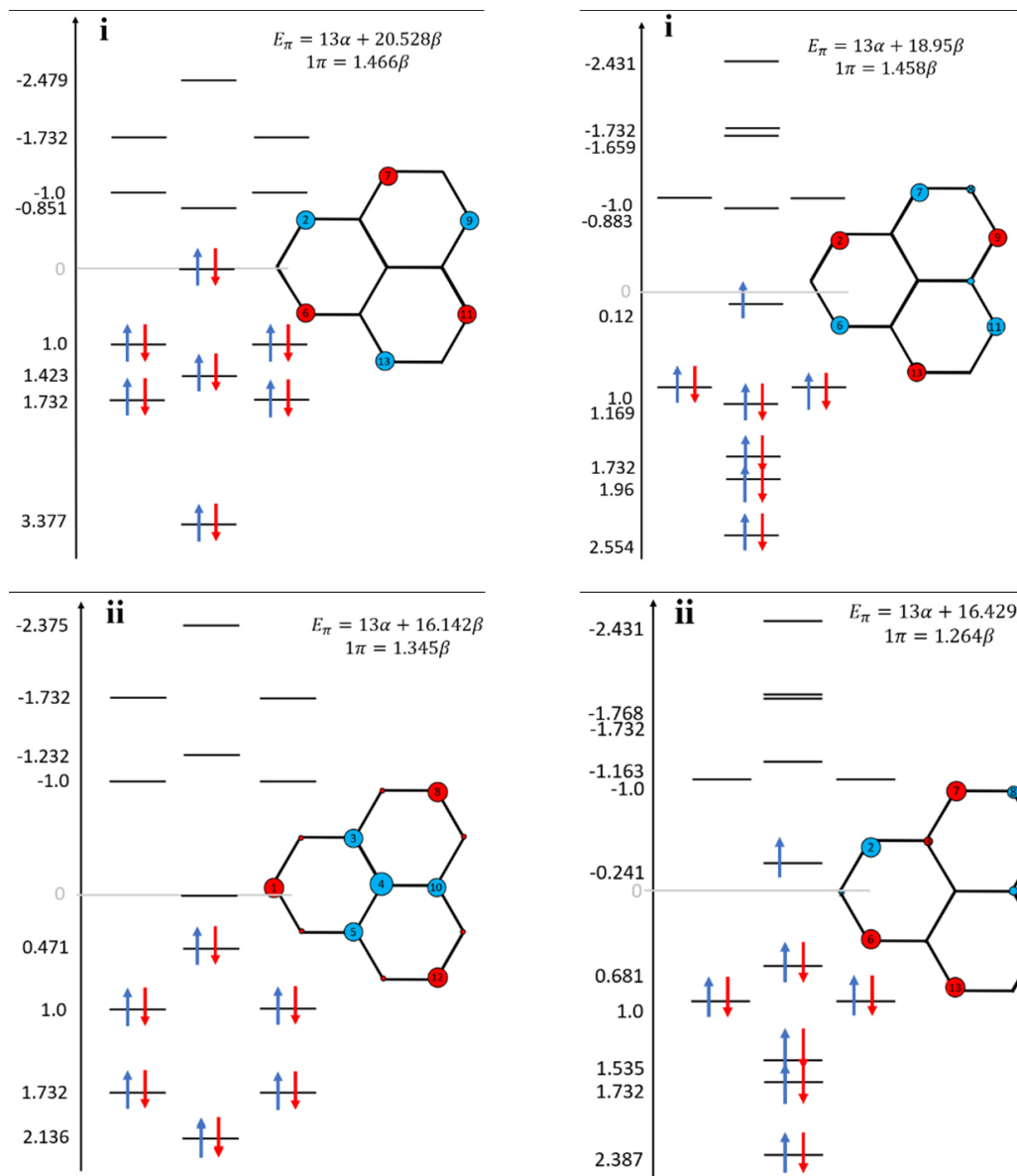

(a)

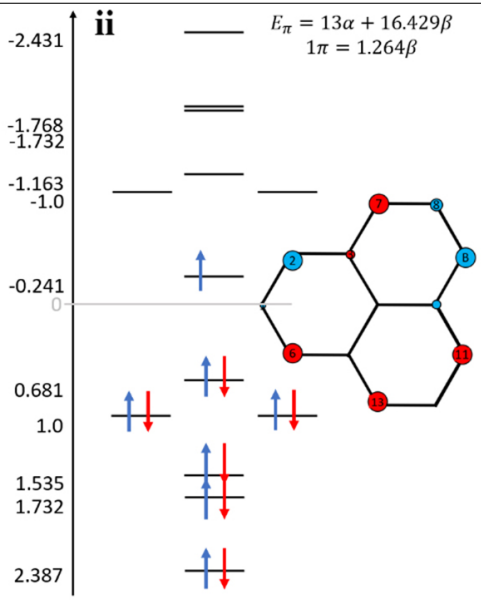

(b)
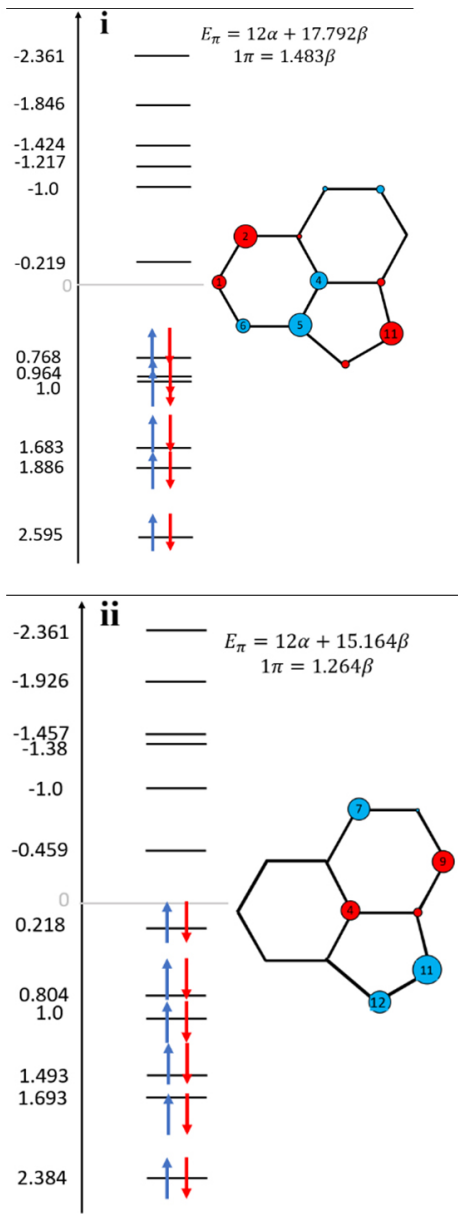

(c)

Figure 7. Energy levels of phenalene doped with (i) nitrogen and (ii) boron and (a) graphitic, (b) pyridinic, and (c) pyrrolic dopant sites

Table 4. Number of $\pi$ electrons, total energy, average $\pi$-electron energy, and gap energy of bare phenalene and its N- or B-doped molecule

\begin{tabular}{|c|c|c|c|c|c|}
\hline Dopant Type/Position & Number of $\pi$ electron & $\begin{array}{c}h_{X} \text { and } \\
k_{X Y} \text { values }\end{array}$ & Total energy & $\pi$-electron energy & Gap energy \\
\hline Bare phenalene & 13 & $h_{C}=0.00 ; k_{C C}=-1.00$ & $13 \alpha+17.826 \beta$ & $1.371 \beta$ & $1.000 \beta$ \\
\hline $\mathrm{N}$-graphitic $\left(\mathrm{C}_{12} \mathrm{NH}_{9}\right)$ & 14 & $\begin{array}{l}h_{N}=-1.00 ; k_{C N}=-0.90 \\
h_{X}=1.47, k_{C N}=-1.30^{*}\end{array}$ & $\begin{array}{l}13 \alpha+18.704 \beta \\
13 \alpha+20.528 \beta\end{array}$ & $\begin{array}{l}1.336 \beta \\
1.466 \beta\end{array}$ & $\begin{array}{c}0.65 \beta \\
0.851 \beta\end{array}$ \\
\hline $\mathrm{N}$-pyridinic $\left(\mathrm{C}_{12} \mathrm{NH}_{9}\right)$ & 13 & $\begin{array}{c}h_{N}=-0.40 ; k_{C N}=-1.00 \\
h_{N}=-0.83 ; k_{C N}=-1.06^{*}\end{array}$ & $\begin{array}{c}13 \alpha+18.264 \beta \\
13 \alpha+18.95 \beta\end{array}$ & $\begin{array}{l}1.405 \beta \\
1.458 \beta\end{array}$ & $\begin{array}{c}0.99 \beta \\
1.003 \beta\end{array}$ \\
\hline N-pyrrolic $\left(\mathrm{C}_{11} \mathrm{NH}_{8}\right)$ & 12 & $\begin{array}{c}h_{N}=-0.40 ; k_{C N}=-1.00 \\
h_{N}=-0.83, k_{C N}=-1.06^{*}\end{array}$ & $\begin{array}{l}12 \alpha+17.082 \beta \\
12 \alpha+17.792 \beta\end{array}$ & $\begin{array}{l}1.424 \beta \\
1.483 \beta\end{array}$ & $\begin{array}{l}0.973 \beta \\
0.987 \beta\end{array}$ \\
\hline B-graphitic $\left(\mathrm{C}_{12} \mathrm{BH}_{9}\right)$ & 12 & $h_{B}=1.00, k_{C B}=-0.70$ & $13 \alpha+16.142 \beta$ & $1.345 \beta$ & $0.471 \beta$ \\
\hline B-pyridinic $\left(\mathrm{C}_{12} \mathrm{BH}_{8}\right)$ & 13 & $h_{B}=1.00, k_{C B}=-0.70$ & $13 \alpha+16.429 \beta$ & $1.264 \beta$ & $0.759 \beta$ \\
\hline B-pyrrolic $\left(\mathrm{C}_{11} \mathrm{BH}_{8}\right)$ & 12 & $h_{B}=1.0, k_{C B}=-0.70$ & $12 \alpha+15.164 \beta$ & $1.264 \beta$ & $0.677 \beta$ \\
\hline
\end{tabular}

*using the experimental $\alpha$ and $\beta$ values from the smallest squares out of UV spectra.

relationships. Ultimately, this study can be supported with further analysis based on advanced calculations to determine various factors such as the optimum dopant amount needed for a specific application.

\section{CONCLUSIONS}

This study investigates the molecular orbital and energy of phenalene/phenalenyl and nitrogen or boron-doped phenalene systems by employing the Hückel method using the HMO software. The delocalization of $\pi$ electrons among carbon atoms at the side position and the radical character of phenalene were observed, which are in good agreement with the findings of previous experimental and theoretical studies. Both the type of dopant and its position in the molecule strongly influence the number of $\pi$ electrons, molecular orbital energy, and properties of the system. Graphitic and pyrrolic dopant types eliminate the radical properties of the molecule, while the pyridinic type of dopant preserves them. More energetic $\pi$ electrons are observed for $\mathrm{N}$-doped phenalene, while lower gap energy is found for B-doped phenalene. The lower energy gap is essential, for example, because it results in stronger photoluminescence spectra. This study elucidates the effect of different dopant on the properties of phenalene molecule. More reliable results and better understanding 
can be achieved through advanced computation methods and upon dopant concentration variation.

\section{ACKNOWLEDGEMENTS}

This paper is dedicated to the late Professor T. M. On for his memorable contributions to the physics and chemistry of conjugated polymers at the Physics Department of the Institut Teknologi Bandung. We would like to thank Editage (www.editage.com) for English language editing.

\section{REFERENCES}

1. Garza, C. G. V.; Olmedo, E. M.; Fomine, S.; Comput. Theor. Chem. 2019, 1151, 12.

2. Permatasari, F. A.; Aimon, A. H.; Iskandar, F.; Ogi, T.; Okuyama, K.; Sci. Rep. 2016, 6, 1.

3. Permatasari, F. A.; Fukazawa, H.; Ogi, T.; Iskandar, F.; Okuyama, K.; ACS Appl. Nano Mater. 2018, 1, 2368.

4. Reid, B. D. H.; Q. Rev. Chem. Soc. 1965, 19, 274.

5. Zoellner, J. M.; Zoellner, R. W.; J. Mol. Struct.: THEOCHEM 2008, 863, 50

6. Morita, Y.; Nishida, S.; In Stable Radicals: Fundamentals and Applied Aspects of Odd-Electron Compounds; Hicks, R. G., ed.; Wiley: New York, 2010, ch. three.

7. Uchida K.; Kubo, T.; J. Synth. Org. Chem. 2016, 74, 1069.

8. Sandoval-Salinas, M. E. A.; Carreras, A.; Casanova, D.; Phys. Chem. Chem. Phys. 2019, 21, 9069.

9. Schneiker, A.; Csonka, I. P.; Tarczay, G.; Chem. Phys. Lett. 2020, 743, 137183.
10. Lazzeretti, P.; Phys. Chem. Chem. Phys. 2004, 6, 217.

11. Koutentis, P. A.; Chen, Y.; Cao, Y.; Best, T. P.; Itkis, M. E.; Beer, L.; Oakley, R. T.; Cordes, A. W.; Brock, C. P.; Haddon, R. C.; J. Am. Chem. Soc. 2001, 123, 3864.

12. Itkis, M. E.; Chi, X; Cordes, A. W.; Haddon, R. C.; Science 2002, 296, 1443.

13. Cyrański, M. K.; Havenith, R. W. A.; Dobrowolski, M. A.; Gray, B. R.; Krygowski, T. M.; Fowler, P. W.; Jenneskens, L. W.; Chem. - Eur. J. 2007, 13, 2201.

14. Bredas, J. L.; Mater. Horiz. 2014, 1, 17.

15. Baerends, E. J.; Gritsenko, O. V.; Van Meer, R.; Phys. Chem. Chem. Phys. 2013, 15, 16408.

16. Hückel, E.; Zeitschrift für Physik 1931, 70, 204.

17. Hückel, E.; Zeitschrift für Physik 1931, 72, 310.

18. Hückel, E.; Zeitschrift für Physik 1932, 76, 628.

19. Hückel, E.; Zeitschrift für Physik 1933, 83, 632.

20. Yates, K.; Hückel molecular orbital theory, Academic Press: New York, 2012.

21. Kutzelnigg, W.; J. Comput. Chem. 2007, 28, 25.

22. HMO Software, https://hueckel-molecular-orbital-hmo.soft112.com, accessed in April 2021

23. Rauk, A.; Orbital Interaction Theory of Organic Chemistry, $2^{\text {nd }}$ ed., Wiley: New York, 2001.

24. Riera-Galindo, S.; Biroli, A. O.; Forni, A.; Puttisong, Y.; Tessore, F.; Pizzotti, M.; Pavlopoulou, E.; Solano, E.; Wang, S.; Wang, G.; Ruoko, T. P.; Chen, W. M.; Kemerink, M.; Berggren, M.; di Carlo, G.; Fabiano, S.; ACS Appl. Mater. Interfaces 2019, 11, 37981. 